\title{
Choice of antibiotics in management of acute osteomyelitis and acute septic arthritis in children
}

\author{
SYDNEY NADE
}

From the Department of Surgery, University of Sydney, Royal North Shore Hospital of Sydney, and Royal Alexandra Hospital for Children, Sydney

SUMMARY A survey of 158 children with acute haematogenous osteomyelitis, and of 94 children with acute septic arthritis over an 8-year period was made to determine which bacteria cause these infections. In the osteomyelitis group the organism most frequently detected was Staphylococcus aureus (74\% of cases). In $16 \%$ of cases streptococci were found. Staph. aureus was also the most frequently grown organism in cases of acute septic arthritis (55\% of cases), but Haemophilus influenzae accounted for $24 \%$ of positive cultures.

On the basis of the survey it is the current practice of the author to use a combination of methicillin or cloxacillin and penicillin for acute haematogenous osteomyelitis, and methicillin or cloxacillin and ampicillin for acute septic arthritis. The choice of antibiotics is vitally important as treatment must start before the results of culture are known. Repeated evaluation of trends in the pattern of causative organisms is strongly recommended, in order to be aware of changing sensitivity of organisms to antibiotics.

Before the advent of antibiotics acute haematogenous osteomyelitis and acute septic arthritis were serious diseases with high morbidity and significant mortality rates (Bick, 1968). Early diagnosis, which is clinical, and administration of appropriate antibiotics may thwart the infection and its sequelae.

During the past 35 years the rate of development of antibiotics and their effective use have altered the clinical course of these diseases. Nevertheless, the emerging resistance patterns of micro-organisms, particularly staphylococci, to antibiotics, have indicated the importance of the accurate recording of bacteriological data. Continuing studies would show microbiological trends which might dictate the revision of policy concerning antibiotic therapy as organisms change their character.

The principle advocated by Gilmour (1962) and again by Blockey (1971) that there are no indications for urgent surgical intervention early in the course of acute osteomyelitis is supported. Despite this, the contention that osteomyelitis is a 'medical' disease to be treated by antibiotics alone and not by surgery is alarming to many orthopaedic surgeons (Jones, 1971). If it is so managed, it should be by a surgeon who is prepared to operate if there are indications for surgery. Currently these are held to be (1) the

Received 16 February 1977 formation of pus (as judged clinically); (2) a period of more than $\mathbf{4 8}$ hours having elapsed since the onset of symptoms (when bone death or articular cartilage destruction is established); (3) failure to gain rapid clinical improvement after conservative management in hospital with fluid and electrolyte replacement, adequate effective antibiotic therapy, and immobilization of the affected parts.

To determine the most appropriate antibiotic or combination of antibiotics it is important to study the causative organisms and their antibiotic sensitivities separately for different geographical areas, as findings reported from one centre do not necessarily apply to any other.

This survey included 158 patients with a diagnosis of acute osteomyelitis, and 94 with acute septic arthritis admitted to three Sydney hospitals during the years 1968 to 1975 , who were reviewed from case records. Of the total, 208 children were admitted to two children's hospitals and 44 to a general hospital.

\section{Review of case records}

Acute osteomyelitis. A clinical diagnosis of acute osteomyelitis was made in 158 cases ( 95 boys, 63 girls), and this was proved by bacterial culture in 92 . The femur and tibia were the bones most commonly affected and in 11 children more than one bone was 
affected. Distribution and frequency of bone involvement are given in Table 1.

Table 1 Bones affected in acute osteomyelitis

\begin{tabular}{ll}
\hline Bone & No. of cases \\
\hline Femur & 40 \\
Tibia & 39 \\
Foot bones & 22 \\
Humerus & 12 \\
Fibula & 8 \\
Pelvis & 6 \\
Spine & 5 \\
Other bones* & 15 \\
More than one & 11 \\
\hline
\end{tabular}

*Clavicle 3, ribs 3, sternum 2, radius 2, patella 2, skull 2, maxilla 1.

The age distribution is shown in Fig. 1. $25 \%$ of the children were under the age of one year, and 62 children $(39 \%)$ were under the age of 3 . Different authors have shown a varying age incidence for this disease (Gilmour, 1962; Trueta, 1968; Blockey and Watson, 1970), with the mean age somewhat higher than in this group.

Of the 158 children, blood for culture was taken from 97 only. Organisms were grown from $44(45 \%)$ of these specimens. Aspirates from suspected areas were taken in 28 cases and organisms were grown in $17(60 \%)$. Aspiration, preferred by some as a means of therapeutic decompression, should probably be used more frequently as a diagnostic aid; Jones (1971) found that in one-sixth of a series of 60 cases, pus was found only in the bone and not under the periosteum. Open operation was performed in 71 cases, and in 58 of these $(82 \%)$ pus was found; from 49 of those specimens an organism was grown, from 9 none were grown. Of those 9, 4 were from patients who had been taking antibiotics before admission to hospital.

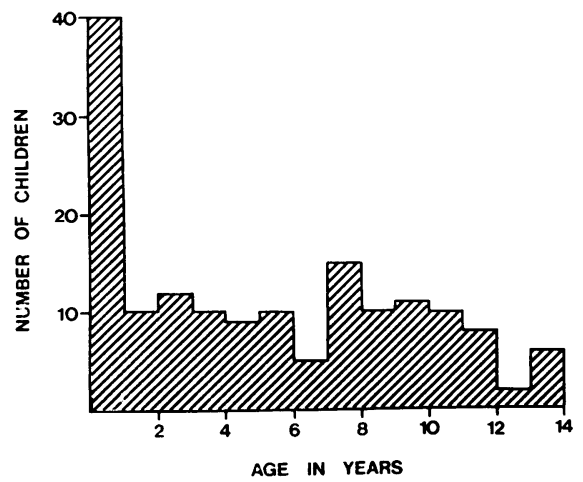

Fig. 1 Age distribution of 158 children with acute haematogenous osteomyelitis, 1968-1975.
Organisms grown on culture and their frequency are given in Table 2.

Table 2 Organisms grown on culture in 158 cases of acute osteomyelitis

\begin{tabular}{lcr}
\hline & Frequency & \\
\cline { 2 - 3 } Organism & No. & $\%$ \\
\hline Staphylococcus aureus & 68 & 74 \\
Streptococcus pyogenes & 8 & 9 \\
Streptococcus pneumoniae & 5 & 5 \\
Streptococcus haemolyticus & 2 & 2 \\
Haemophilus influenzae & 3 & 3 \\
Escherichia coli & 2 & 2 \\
Bacillus proteus & 2 & 2 \\
Salmonella & 2 & 1 \\
Moraxella & 1 & \\
Pus found-no organisms & 10 & 2 \\
\hline
\end{tabular}

Staphylococcus aureus (or pyogenes) was grown in $74 \%$ of cases in which bacteria were cultured and Streptococcus pyogenes, Strep. haemolyticus, or Strep. pneumoniae in $16 \%$. In other reported series the incidence of Staph. aureus has varied from $75 \%$ to $96 \%$ (Winters and Cahen, 1960; Gilmour, 1962; Meyer et al., 1965; Green, 1967; Blockey and McAllister, 1972).

Acute septic arthritis. 94 patients ( 58 boys, 36 girls) with a clinical diagnosis of acute septic arthritis were studied. Bacteriological proof of diagnosis was obtained in 59. Joints which were affected and the frequency of involvement are shown in Table 3.

Table 3 Joints affected in acute septic arthritis

\begin{tabular}{ll}
\hline Joint & Frequency \\
\hline Knee & $37^{*}$ \\
Hip & 31 \\
Ankle & $18^{*}$ \\
Shoulder & 4 \\
Elbow & 4 \\
Metatarsophalangeal & 2 \\
Sternoclavicular & $1^{*}$
\end{tabular}

*Includes multiple joints (both knees, knees and ankles, knee and sternoclavicular).

Distribution of ages is shown in Fig. 2 and it is notable that 42 children $(45 \%)$ were under the age of 3 years. Blood for culture was taken from only 51 patients, and in $20(39 \%)$ the results were positive. Aspirates from joints wese taken in 48 cases and in 16 of these $(65 \%)$ organisms were grown. In 48 cases surgery was performed and pus was found in 45 ; in 39 of these organisms were grown from the pus (Table 4). 


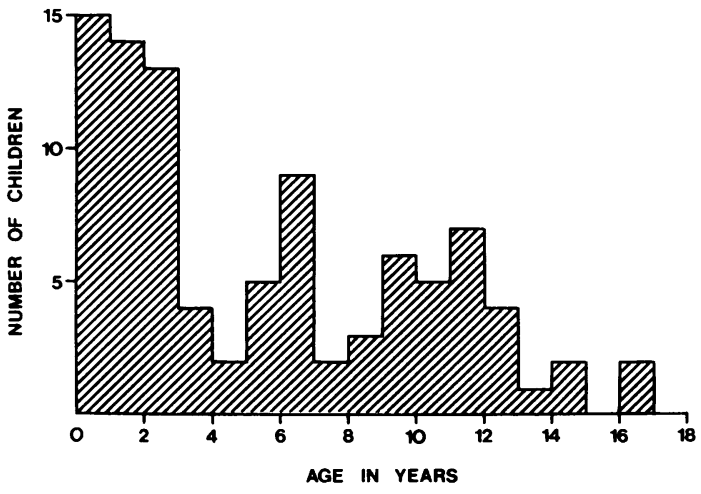

Fig. 2 Age distribution of 94 children with acute septic arthritis, 1968-1975.

Table 4 Organisms grown on culture (58) in 94 cases of septic arthritis

\begin{tabular}{lll}
\hline & \multicolumn{2}{l}{ Frequency } \\
\cline { 2 - 3 } Organism & No. of cases & $\%$ \\
\hline Staphylococcus aureus & 32 & 55 \\
Staphylococcus albus & 1 & \\
Streptococci $\beta$-haemolytic & 7 & 24 \\
Haemophilus influenzae & 14 & \\
Escherichia coli & 1 & \\
Bacillus proteus & 1 & \\
Clostridium welchii* & 1 & \\
Serratia marcescens & 1 & \\
Pus found-no organism & 6 & \\
\hline
\end{tabular}

*Associated with intra-articular foreign body.

Staphylococci accounted for $57 \%$ and $\mathrm{H}$. influenzae for $24 \%$ of the bacteria isolated. Streptococci were not found in the first 5 years of the study, but occurred in 7 cases during the last 3 years. Table 5 shows the antibiotic sensitivities of the various organisms found in both osteomyelitis and septic arthritis.

\section{Discussion}

The incidence of chronic osteomyelitis bears a direct relationship to the duration between onset of symptoms of acute osteomyelitis and the time of starting antibiotic therapy. This has been confirmed by D. C. Davidson (personal communication, 1974) in a study similar to this at Adelaide Children's Hospital. The critical time was 3 days, after which there was a marked rise in incidence of the chronic condition.

The antibiotic sensitivity of cultured organisms from blood, bone, joint, or soft tissue aspirates or from pus found at surgery usually takes about 2 days to develop. Examination of specimens by Gram staining may give an earlier clue to the causative organism. However, antibiotics are usually given on a 'best guess' basis until accurate results are available, and it is important that antibiotics be started as soon as diagnostic aspirates have been drawn.

It is my personal practice to use parenterally, in adequate dose, the following antibiotics.

(a) For acute osteomyelitis, methicillin or cloxacillin and penicillin; (b) for acute septic arthritis, methicillin or cloxacillin and ampicillin. These combinations give the most effective cover in acute osteomyelitis for staphylococci and streptococci (which together account for $90 \%$ of infections), and in acute septic arthritis for staphylococci and $\boldsymbol{H}$. influenzae, which account for $79 \%$ of infections.

Several combinations of antibiotics have been recommended for treatment of acute osteomyelitis, notably benzyl penicillin and cloxacillin (Blockey and Watson, 1970) and fucidic acid and erythromycin (Blockey, 1971; Blockey and McAllister, 1972). The rationale for the use of the latter combination was the predicted emergence of strains of staphylococci resistant to methicillin (Rountree and Vickery, 1973). Although these organisms exist and are indeed becoming more frequent, they appear to produce nosocomial infection, and no case of acute osteomyelitis due to a methicillin-resistant staphylococcus has yet been reported in Australia (S. W. Williams, 1973, personal communication). Lincomycin and clindamycin may also be useful for those patients who are intolerant of penicillins (Tolhurst et al., 1972). Similarly, ampicillin-resistant $H$. influenzae organisms exist, but none have caused a reported case of acute septic arthritis.

At the major children's hospital involved in this study, during the same period of time, organisms have been grown from patients with bacteraemia on

Table 5 Antibiotic sensitivities of organisms

\begin{tabular}{|c|c|c|c|c|c|c|c|c|}
\hline \multirow[b]{2}{*}{ Organism } & \multicolumn{8}{|c|}{ Percentage of organisms sensitive to } \\
\hline & Penicillin & Sulphonamide & Erythromycin & Ampicillin & $\begin{array}{l}\text { Methicillin/ } \\
\text { cloxacillin }\end{array}$ & Kanamycin & Chloramphenicol & Tetracycline \\
\hline Staph. aureus & 18 & 50 & 95 & & 100 & 100 & 96 & 93 \\
\hline H. influenzae & & 46 & & 100 & & 100 & 92 & 100 \\
\hline Streptococci & 100 & & 100 & & & & 100 & \\
\hline
\end{tabular}


776 occasions. Of these infections, $134(17 \%)$ were staphylococcal; 21 of these patients had osteomyelitis and 10 had septic arthritis. These diseases together are the most common companions of staphylococcal bacteraemia. Early and adequate treatment can prevent multiple foci of infection. At present the antibiotic sensitivity patterns of the organisms which cause acute haematogenous osteomyelitis and acute septic arthritis are not significantly different from those organisms grown generally in hospital populations. Because acute osteomyelitis and acute septic arthritis are uncommon diseases, a continuing study of the organisms grown from bacteraemias should indicate the changing pattern of antibiotic sensitivity.

The natural history of acute septic arthritis is also changing. In 1966, Nelson and Koontz reported that $H$. influenzae was becoming a common causative organism in acute septic arthritis. Based on a bacteriological study similar to this, they were the first to suggest a rational treatment regimen. They also commented on the relation of the child's age to the organism found, stating that if the infant were under 6 months of age the most likely organisms were staphylococci or Gram-negative enteric pathogens; between 6 months and 2 years of age, staphylococci or $\boldsymbol{H}$. influenzae; and over 2 years of age, staphylococci. The reason for this remains unknown but it is confirmed in the present study. Examination of a Gram-stain smear of aspirates from children under 2 years could therefore be useful in determining whether the most likely organism was a staphylococcus, an enteric pathogen, or $H$. influenzae. As more and more cases of acute osteomyelitis are being treated by antibiotics alone, the yield of organisms cultured will decrease. Because bacteriological confirmation and diagnosis and culture for antibiotic sensitivity takes so much time, antibiotics cannot be withheld until data are obtained. Furthermore, organisms are grown in only about half the cases. The most appropriate antibiotics for early treatment are therefore given on a 'best guess' basis. However, if studies such as this one are performed regularly it will be possible to determine appropriate antibiotic treatment, and the use of electronic data processing methods, as in this study, will permit frequent and rapid reprocessing of data which may indicate the changing patterns in bacteriology and antibiotic sensitivity of a common infecting organism.
I thank Mr. George Nade, who developed the computer program (written so that repeated analyses at different times and involving multiple centres, can be performed); Dr. F. W. Robertson and Dr. G. Kalnins who helped procure data from case notes; Dr. David Dorman, microbiologist at the Royal Alexandra Hospital for Children who provided microbiological data; and Professor T. K. F. Taylor for helpful discussion.

\section{References}

Bick, E. M. (1968). Source Book of Orthopaedics, 2nd ed. Hafner, New York.

Blockey, N. J. (1971). Conservative management of acute osteomyelitis. Proceedings of the Royal Society of Medicine, 64, 1199.

Blockey, N. J., and McAllister, T. A. (1972). Antibiotics in acute osteomyelitis in children. Journal of Bone and Joint Surgery, 54B, 299-308.

Blockey, N. J., and Watson, J. T. (1970). Acute osteomyelitis in children. Journal of Bone and Joint Surgery, 52B, 77-87.

Gilmour, W. N. (1962). Acute haematogenous osteomyeltis. Journal of Bone and Joint Surgery, 44B, 841-853.

Green, J. H. (1967). Cloxacillin in the treatment of acute osteomyelitis. British Medical Journal, 2, 414-416.

Jones, G. B. (1971). Place of surgery in treatment of acute haematogenous osteomyelitis. Proceedings of the Royal Society of Medicine, 64, 1200-1201.

Meyer, T. L., Kieger, A. B., and Smith, W. S. (1965). Antibiotic management of staphylococcal osteomyelitis. Journal of Bone and Joint Surgery, 47A, 285-292.

Nelson, J. D., and Koontz, W. C. (1966). Septic arthritis in infants and children. A review of 117 cases. Pediatrics, 38, 966-971.

Rountree, P. M., and Vickery, A. M. (1973). Further observations on methicillin-resistant staphylococci. Medical Journal of Australia, 1, 1030-1034.

Tolhurst, J. C., Buckle, G., and Williams, S. W. (1972). Chemotherapy with Antibiotics and Allied Drugs. Australian Government Publishing Service, Canberra.

Trueta, J. (1968). Studies of the Development and Decay of the Human Frame. Heinemann, London.

Winters, J. L., and Cahen, I. (1960). Acute hematogenous osteomyelitis. Journal of Bone and Joint Surgery, 42A, 691-704.

Correspondence to Mr. S. Nade, Department of Orthopaedics and Traumatic Surgery, The Royal North Shore Hospital of Sydney, St. Leonards, New South Wales 2065, Australia. 